\section{GW23-e1590 EFFECTS OF AUTOLOGOUS BONE MARROW MONONUCLEAR CELLS TRANSPLANTATION VIA CORONARY ARTERY IN PATIENTS WITH ACUTE MYOCARDIAL INFARCTION ASSESSED BY MRI}

doi:10.1136/heartjnl-2012-302920j.37

Minjie Lu, Lei Song, Shiliang Jiang, Yuejing Yang, Yan Zhang, Gang Yin, Shihua Zhao. Fuwai Hospital \& Cardiovascular Institute, Chinese Academy of Medical Sciences \& Tsinghua University, Peking Union Medical College, National Center for Cardiovascular Diseases

Objectives The aim of this study was to use an 'one-stop' non-invasive imaging examination-MRI to evaluate the feasibility and safety of aBM-MNC transplantation in patients with acute myocardial infarction (AMI) undergoing percutaneous coronary intervention.

Methods We did a randomised, double-blind, placebo-controlled study in 60 patients (male $=43$, female $=17$, age $52.18 \pm 4.98$ years) with AMI. The patients were randomly divided into 2 groups (group A: PCI+ aBM-MNC, group B: PCI only). Preoperative global left ventricular functions and scar tissue were measured by MRI. The therapeutic effects were assessed by MRI 6-month after aBM-MNC transplantation.

Results ALL the patients were treated without major complications. There is no evidence of new ventricular arrhythmia or neoplasia. The LVEF was improved $28.5 \%$ in group $\mathrm{A}$, while $18.4 \%$ in group $B(p<0.01), \mathrm{LVEDV} / \mathrm{m} 2$ and $\mathrm{LVESV} / \mathrm{m} 2$ were decreased by $21.15 \pm 3.96 \mathrm{ml} / \mathrm{m}^{2}$ and $27.14 \pm 4.48$, respectively, which were significantly different from that in group $B(5.85 \pm 6.18 \mathrm{ml}(\mathrm{p}=0.08)$ and $9.18 \pm 4.84(p=0.04))$. The cardiac output $(\mathrm{CO})$, cardiac index (CI) and cardiac mass (CM) didn't show significant difference between the two groups. Compared with group B, aBM-MNC group was associated with no significant reduction in myocardial infarct size $(15.3 \%$ vs $12.7 \%, p=0.51)$.

Conclusions Comprehensive in vivo CMR reveals reversed remodelling and improved systolic function and scar characteristics after aBM-MNC transplantation. PCI+aBM-MNC transplantation can lead to comparable improvements of left ventricle in acute myocardial infraction. 